\title{
Special Collection: Suicide Awareness and Prevention in Psychiatric Education: Introduction and Dedication
}

\author{
Jon A. Lehrmann • Laura Weiss Roberts
}

Received: 2 July 2014 / Accepted: 4 July 2014 / Published online: 1 August 2014

(C) Academic Psychiatry 2014

Charles E. Kubly died as a young man after struggling with severe and recurring depression for many years. Charlie's mother, Billie, and father, Michael, with the full efforts of their family, have responded to this tragedy with passion and tremendous strength, actively and constantly working to improve the lives of those who struggle with depression. They established the Charles E. Kubly Foundation in 2003, and its mission is seeking "to better the lives of those affected by depression by increasing public awareness of the disease and its devastating effects, eliminating the stigma associated with it, supporting suicide prevention programs and promoting improved access to quality mental health resources within communities." Through the Foundation's competitive small grant program, the editor-in-chief, Laura Weiss Roberts, submitted a proposal for an issue of our journal to include a focus on education in psychiatry related to suicide, and this special collection of papers is the result. The editors thank Jon A. Lehrmann, M.D., the Charles E. Kubly Professor of Psychiatry and
Behavioral Medicine of the Medical College of Wisconsin, who worked with us to create this collection.

The Charles E. Kubly Foundation has raised over $\$ 1.3$ million over the past 10 years and uses these funds to support diverse projects. Readers of the journal may wish to learn more by looking at the Foundation's website, http:// charlesekublyfoundation.org/. A few examples of projects they have supported include an art education engagement program for high school students, a mental health summit at a local college, stigma reduction talks at local schools, youth education retreats focusing on helping develop coping skills, and "Train the Trainer" education for correctional officers on depression and suicide prevention. The Charles E. Kubly Foundation produces an annual event in Milwaukee, "Beyond the Blues," where people gather for fun, music, and great food, symbolizing hope and fostering the role of community in addressing depression and its impact.

This special collection in Academic Psychiatry is dedicated to Charlie and all who loved him.

\footnotetext{
J. A. Lehrmann ( $\square)$

Medical College of Wisconsin, Milwaukee, WI, USA

e-mail: jon.lehrmann@va.gov

L. W. Roberts

Stanford University, Stanford, CA, USA
} 